\title{
Surgical and molecular considerations in the treatment of pediatric thalamopeduncular tumors
}

\author{
Ryan P. Lee, BA, ${ }^{1}$ Kimberly A. Foster, MD, ${ }^{2,3}$ Jock C. Lillard, BS, ${ }^{1}$ Paul Klimo Jr., MD, MPH, ${ }^{2-5}$ \\ David W. Ellison, MD, PhD, ${ }^{6}$ Brent Orr, MD, PhD, ${ }^{6}$ and Frederick A. Boop, MD ${ }^{2-5}$ \\ ${ }^{1}$ College of Medicine, University of Tennessee Health Science Center; ' 2 Department of Neurosurgery, Le Bonheur Children's \\ Hospital; ${ }^{3}$ Division of Neurosurgery, St. Jude Children's Research Hospital; ${ }^{4}$ Department of Neurosurgery, University of \\ Tennessee Health Science Center; ${ }^{5}$ Semmes-Murphey Neurologic \& Spine Institute; and ${ }^{6}$ Department of Pathology, St. Jude \\ Children's Research Hospital, Memphis, Tennessee
}

OBJECTIVE Thalamopeduncular tumors are a group of pediatric low-grade gliomas that arise at the interface of the thalamus and brainstem peduncle. They typically occur within the first 2 decades of life, presenting with progressive spastic hemiparesis. Treatment strategies, including surgical intervention, have varied significantly. The authors present their experience in the treatment of 13 children, ages 2-15 years, with non-neurofibromatosis-related pilocytic astrocytomas located in the thalamopeduncular region.

METHODS Between 2003 and 2016, 13 children presenting with progressive spastic hemiparesis due to a pilocytic astrocytoma at the interface of the thalamus and cerebral peduncles were identified. Medical records were reviewed retrospectively for clinical, radiological, pathological, and surgical data. Formalin-fixed, paraffin-embedded tissue was obtained for 12 cases and tested for KIAA1549-BRAF fusion and BRAF V600E point mutation.

RESULTS On preoperative diffusion tensor imaging tractography (performed in 12 patients), the ipsilateral corticospinal tract was displaced laterally in 1 case (8.3\%), medially in 1 case (8.3\%), anterolaterally in 10 cases (83\%), and posteriorly in no cases. Ten patients underwent resection via a transtemporal, transchoroidal approach, which was chosen to avoid further damage to motor function in cases of tumors that caused anterolateral or medial corticospinal tract displacement. With this approach, complications included hemianopia, oculomotor palsy, and tremor at a rate of 50\%. Among the 12 patients with obtainable follow-up (mean 50.9 months), none received adjuvant therapy, and only 2 (17\%) experienced recurrence or progression. KIAA1549-BRAF fusions were present in 10 cases (83\%), while BRAF V600E was absent $(0 \%)$. The 2 fusion-negative tumors had clinical features atypical for the series, including multi-focality and infiltration.

CONCLUSIONS Transcortical, transchoroidal resection of thalamopeduncular tumors through the middle temporal gyrus allows for a high rate of gross-total resection and cure. Diffuse tensor tractography is a critical component of the preoperative planning process to determine the location of white matter tracts in proximity. Molecular status may correlate with clinical features, and the presence of BRAF lesions offers an additional target for future novel therapeutics.

https://thejns.org/doi/abs/10.3171/2017.4.PEDS16668

KEY WORDS thalamic; thalamopeduncular; tumor; pediatric; pilocytic astrocytoma; BRAF; low-grade glioma; genetics; diffusion tensor imaging; tractography; oncology

$\mathrm{T}$ HE thalamopeduncular syndrome of childhood is a clinical presentation of progressive spastic hemiparesis caused by a pilocytic astrocytoma at the interface of the thalamus and cerebral peduncle. Puget et al. ${ }^{31}$ introduced this term to the nomenclature in 2011, but optimal treatment and appropriate subclassification of these pediatric tumors remains a moving target. $2,5,7,8,10,11,13,15-17,20,22,24-$
26,28,29,34,38-43,47 While many therapeutic strategies have been considered, we previously reported success in achieving disease control and frequent cure after gross-total resection (GTR) performed via a transcortical approach through the middle temporal gyrus. ${ }^{4}$ In conjunction with frameless stereotaxy and contemporary microneurosurgical technique, the use of tractography via diffuse tensor imaging was crit-

ABBREVIATIONS CST = corticospinal tract; DTI = diffusion tensor imaging; GTR = gross-total resection; LGG = low-grade glioma; MAPK = mitogen-activated protein kinase; PCGP = Pediatric Cancer Genome Project; RT-PCR = reverse transcription polymerase chain reaction.

SUBMITTED December 5, 2016. ACCEPTED April 10, 2017.

INCLUDE WHEN CITING Published online July 7, 2017; DOI: 10.3171/2017.4.PEDS16668. 
ical to surgical planning to avoid damaging adjacent white matter structures. For a lesion once considered by many to be inoperable or minimally amenable to resection, we were able to achieve a complete resection with an acceptable rate of operative morbidity, and we argued for a central role of surgical intervention in its treatment.

Given the low incidence of these tumors, nuances in management for this region of the brain are still being elucidated while awaiting larger sample sizes and better subclassification. Therefore, in the present report, we provide one of the most robust series yet reported by adding 4 additional patients, 5 additional years of follow-up, and a molecular analysis. The aims of this study were to better define the presentation and disease course of thalamopeduncular tumors, to further evaluate the optimal role and method of surgical intervention, and to explore the molecular characteristics of these tumors that may correlate with clinical features and provide novel therapeutic targets.

\section{Methods}

\section{Patient Cohort}

Between 2003 and 2014, 13 children were identified who presented with thalamopeduncular syndrome of childhood, characterized as a progressive spastic hemiparesis caused by a tumor at the interface of the thalamus and cerebral peduncle. Children were referred to the combined brain tumor program at St. Jude Children's Research Hospital and Le Bonheur Children's Hospital in Memphis; many were transferred after undergoing varying degrees of diagnosis and treatment elsewhere. Both retrospective and prospective data collection was approved by the Institutional Review Boards of the University of Tennessee Health Science Center and St. Jude Children's Research Hospital. Only tumors of pilocytic astrocytoma histology were included, which excluded a patient with fibrillary astrocytoma who presented with this clinical syndrome. Patients with neurofibromatosis were also excluded. Patients 1 through 9 in the present series correspond to patients 1 , 2 , and 4 through 10 in our previous case series. ${ }^{4}$ Patient 3 in that previous series was the case of fibrillary astrocytoma that is herein excluded.

\section{Clinical Variables}

As a part of their clinical evaluation and management, detailed MRI information was obtained, as previously detailed. ${ }^{4}$ In all except the first patient, this included tractography achieved via diffusion tensor imaging (DTI) to the planned surgical approach. Parameters of imaging acquisition and analysis have also been detailed previously. ${ }^{4} \mathrm{Im}$ aging studies were retrospectively reviewed, specifically for determining the relationship of the tumor to the corticospinal tract (CST) and other critical adjacent white matter structures. Medical records were reviewed for patient demographics, clinical presentation, treatment parameters, surgical approach, neurological outcome, and disease progression. Only limited details about chemotherapeutic regimens received before the patients presented to our center were obtainable through retrospective review and are noted in the article accordingly. Clinical follow-up was carried through August 1, 2016. For patients without re- cent follow-up data in their medical record, we contacted patient families via telephone in concordance with our IRB-approved protocol.

\section{Molecular Testing}

Formalin fixed, paraffin-embedded tissue samples were obtained and tested for the presence of $B R A F$ V600E point mutation and KIAA1549-BRAF fusion in 12 of the 13 cases. KIAA1549-BRAF fusion status was determined first by fluorescence in situ hydridization (FISH) for the 7q34 duplication, then confirmed by reverse transcription polymerase chain reaction (RT-PCR) and Sanger sequencing when possible. These methods have also been detailed previously. ${ }^{46} B R A F$ V600E status was determined by RTPCR and Sanger sequencing, using previously validated and published primers. ${ }^{46}$ Additional molecular genetic data were available for 6 cases from the Pediatric Cancer Genome Project (PCGP) and are included with permission. ${ }^{46}$

\section{Study Questions}

1. How do pilocytic astrocytomas respond to standard treatment modalities of chemotherapy, radiotherapy, and surgical resection?

2. What is the optimal surgical approach for achieving GTR while minimizing surgical morbidity and maximizing progression-free survival?

3. How do molecular genetics correlate with disease behavior and what opportunities for novel therapeutics does this analysis provide?

\section{Results \\ Clinical Presentation}

All children in this series presented with varying degrees of progressive spastic hemiparesis, resulting from mass effect of the tumor (Table 1). Ages ranged from 2 to 15 years (median 7 years), with 5 female and 8 male patients. Headache was a notable contributing symptom in 4 patients $(31 \%)$, and $1(7.7 \%)$ patient experienced nausea and vomiting from raised intracranial pressure due to hydrocephalus. Six patients had not undergone any intervention prior to presentation. One patient had previously undergone shunt placement due to large tumor size and symptomatic hydrocephalus. Two of the patients had received a diagnostic biopsy and were subsequently referred to our center. Two patients had undergone biopsies and treatment with chemotherapy (carboplatin and vincristine) but were then referred to our center after progression of disease. Another 2 patients had undergone pre-referral subtotal resections, followed by chemotherapy and subsequent disease progression. In total, all 4 patients who underwent chemotherapy prior to presentation to our program experienced disease progression that required definitive surgical management.

\section{Relationship to CSTs and Surrounding Structures}

On retrospective review of preoperative DTI tractography studies obtained in the available 12 cases (not performed in patient 1), the ipsilateral CST was displaced laterally in 1 case (8.3\%), medially in 1 case, and anterolater- 
TABLE 1. Clinical characteristics and presentation*

\begin{tabular}{|c|c|c|c|c|c|c|}
\hline Pt No.† & Sex & Age (yrs) & Presentation & Prior Management & Prior Surgical Approach & Result of Prior Management \\
\hline $1(1)$ & M & 7 & Hemiparesis & - & - & - \\
\hline $2(2)$ & $\mathrm{F}$ & 12 & Hemiparesis & Resection \& chemo & Frontal transcortical & STR, progression \\
\hline $3(4)$ & $\mathrm{F}$ & 8 & Hemiparesis & - & - & - \\
\hline $4(5)$ & $\mathrm{F}$ & 10 & Hemiparesis & Bx \& chemo & - & Progression \\
\hline $5(6)$ & M & 13 & Hemiparesis & $\mathrm{Bx}$ & - & - \\
\hline $6(7)$ & $\mathrm{F}$ & 3 & Hemiparesis & Resection \& CT & Frontal transcortical & STR, progression \\
\hline $7(8)$ & $\mathrm{F}$ & 6 & Hemiparesis & Bx & - & - \\
\hline $8(9)$ & $M$ & 10 & Hemiparesis, OP & Bx \& chemo & - & Progression \\
\hline $9(10)$ & M & 15 & Hemiparesis, seizure & - & - & - \\
\hline 10 & $M$ & 6 & Hemiparesis, $\mathrm{HH}$ & - & - & - \\
\hline 11 & $M$ & 6 & Hemiparesis, HCP & - & - & - \\
\hline 12 & $M$ & 5 & Hemiparesis, HCP & VP shunt & - & - \\
\hline 13 & $M$ & 2 & Hemiparesis & - & - & - \\
\hline
\end{tabular}

$\mathrm{Bx}=$ biopsy; chemo = chemotherapy; $\mathrm{HA}$ = headache; $\mathrm{HCP}=$ hydrocephalus; $\mathrm{HH}=$ homonymous hemianopsia; $\mathrm{OP}$ = ophthalmoplegia; $\mathrm{Pt}=$ patient; STR = subtotal resection; VP = ventriculoperitoneal; $-=$ not applicable.

* All patients presented with progressive, spastic hemiparesis. A few patients had additional presenting symptoms as noted. All patients treated at another institution with chemotherapy, with or without resection attempts, eventually had progression.

$\dagger$ Number in parentheses is case number from our previous publication.

$\ddagger$ Received combination carboplatin/vincristine.

ally in 10 cases (83\%) (Table 2). In no case was the CST displaced posteriorly. With gradual enlargement of the tumors, they appeared to displace the thalamus superiorly after growing across the ambient cistern. The optic tract was always displaced laterally. In cases where the tumor extended along the length of the peduncle into the posterior fossa, the third cranial nerve was stretched inferiorly.

\section{Surgical Approaches and Outcomes}

The first patient in the series, presenting before we had acquired the DTI/tractography software, experienced worsening hemiparesis after resection (Table 2). Because the tumor had grown into the temporal horn of the lateral ventricle, a transsylvian approach was used and GTR was achieved. The worsening hemiparesis could not be explained by postoperative imaging, but we suspect his motor tracts had been displaced anterolaterally. In subsequent cases, tractography was used to assess the relationship of the CSTs to the tumor in an attempt to avoid this outcome.

The second patient had undergone prior subtotal resec-

TABLE 2. Surgical characteristics and outcomes*

\begin{tabular}{|c|c|c|c|c|c|c|}
\hline Pt No.† & CST Displacement & Our Surgical Approach & Complications & EOR & FU Mos & FU Status \\
\hline $1(1)$ & - & Transsylvian & Worsened paresis & GTR & 147 & Disease free \\
\hline $2(2)$ & Anterolateral & Transsylvian & $\mathrm{HH}, \mathrm{ION}$ & GTR & 83 & Disease free \\
\hline $3(4)$ & Anterolateral & MTG & - & GTR & 96 & Disease free \\
\hline $4(5)$ & Medial & MTG & - & GTR & 0 & - \\
\hline $5(6)$ & Anterolateral & MTG & $3 r d C N$ & GTR & 4 & Disease free \\
\hline $6(7)$ & Lateral & Transfrontal (via old tract) & - & GTR & 46 & Disease free \\
\hline $7(8)$ & Anterolateral & MTG & $3 r d C N$ & GTR & 73 & Disease free \\
\hline $8(9)$ & Anterolateral & MTG & Tremor & STR & 83 & Progression; RT/chemo yielded stable residual \\
\hline $9(10)$ & Anterolateral & MTG & $\mathrm{HH}$ & STR & 21 & Stable residual \\
\hline 10 & Anterolateral & MTG & - & GTR & 11 & Disease free \\
\hline 11 & Anterolateral & MTG then retromastoid & - & GTR & 18 & Disease free \\
\hline 12 & Anterolateral & MTG & - & STR & 11 & Stable residual \\
\hline 13 & Anterolateral & MTG & $\mathrm{HH} \ddagger$ & GTR & 17 & Recurrence; GTR yielded disease-free status \\
\hline
\end{tabular}

$\mathrm{CN}=$ cranial nerve; $\mathrm{EOR}=$ extent of resection; FU = follow-up; ION = intranuclear ophthalmoplegia; $\mathrm{MTG}=$ middle temporal gyrus; $\mathrm{RT}=$ radiation therapy.

* Direction of CST displacement as measured by DTI was determined in all patients except the first. After the first patient, a transcortical approach through the middle temporal gyrus was used to avoid the CSTs unless a previous approach had already been attempted (as in Patients 2 and 6) or a staged resection was necessary (as is Patient 11). Persistent complications from resection at our institution are listed when present. Follow-up duration was calculated as time from surgery to most recent follow-up MRI.

$\dagger$ Number in parentheses is case number from previous publication.

$\ddagger$ Homonymous hemianopsia complication occurred after the second surgery. 
tion at another institution; the surgery had been performed via a frontal transcortical approach that resulted in transient aphasia and spastic hemiparesis. On presentation to our institution, tractography demonstrated a completely disrupted CST, so a transsylvian approach was again used, which allowed for GTR without affecting speech function or worsening the existing hemiparesis.

The third patient presented without having undergone any prior intervention, so tractography was performed for surgical planning. With anterolateral displacement of the CST, a transcortical, transchoroidal approach through the middle temporal gyrus was designed. GTR was achieved without complication and the patient recovered motor function significantly. In the subsequent 10 cases, the CSTs were displaced anterolaterally in 8 , medially in 1 , and laterally in 1. For the anterolaterally and medially displaced cases, a middle temporal gyrus approach was used consistently (Figs. 1 and 2), allowing for GTR in 6 of those 9 (78\%). However, this approach could not be used in the 1 case with lateral displacement (Patient 6). A transfrontal approach had been attempted in Patient 6 at another institution 6 months prior, resulting in subtotal resection (70\%-80\% remaining) and progression despite the surgery and chemotherapy, so the old resection tract was used. GTR was achieved on second resection without complication.

In all, 10 patients have undergone resection transcortically through the middle temporal gyrus. The rate of GTR was $70 \%$ ( 7 of 10) with this approach. Of the 3 cases of subtotal resection, 1 case (Patient 8) had a multifocal tumor that could not be cured surgically, 1 (Patient 9) had infiltrative features and a small residual tumor on postoperative imaging, and 1 (Patient 12 ) had an atypically large tumor for which GTR was not attempted, but a $95 \%$ resection was achieved. Two cases $(20 \%)$ resulted in damage to the third cranial nerve; $2(20 \%)$ suffered damage to the optic tract, resulting in homonymous hemianopia; and 1 $(10 \%)$ resulted in tremor. Five $(50 \%)$ of 10 patients had no complications from the middle temporal gyrus approach. One patient (10\%) had an unexplained ipsilateral ischemic optic neuropathy.

With current appreciation for frequent anterolateral displacement of the CST by these tumors, we retrospectively concluded that the CST was most likely inherently
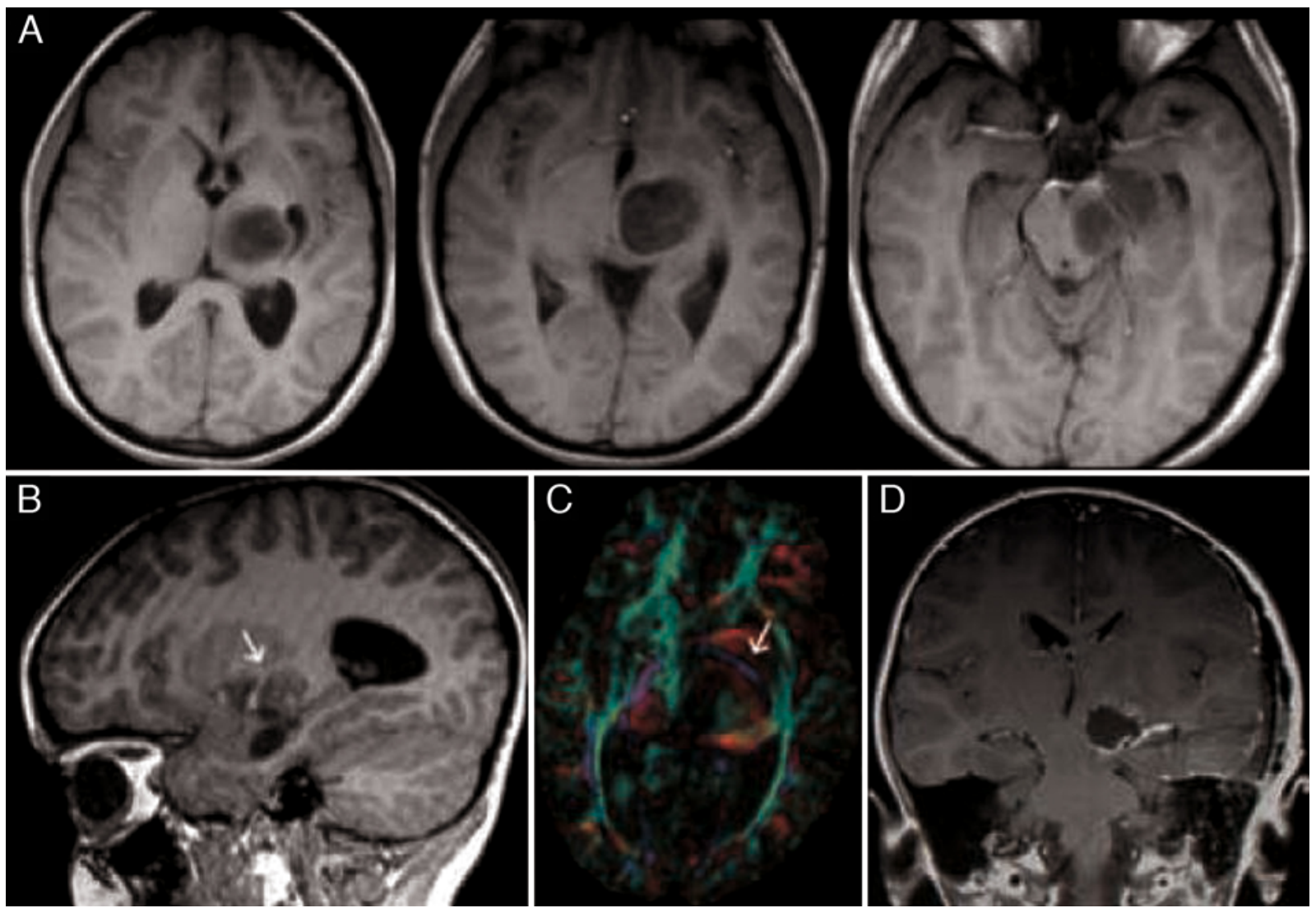

FIG. 1. Representative left thalamopeduncular pilocytic astrocytomas. A: Composite of 3 axial T1-weighted Gd-enhanced MR images demonstrating that the tumor arises from the lateral aspect of the peduncle underneath the thalamus, pushing the normal thalamus superiorly. The thalamic displacement makes a transcallosal approach to the tumor a poor choice, because the surgeon would violate the normal thalamus to reach the tumor. B: The optic tract, a structure that must be carefully avoided when removing the tumor, is deviated superior and lateral to the tumor. The arrow designates the optic tract. C: Axial DT image of the same tumor showing the CSTs deviated anteriorly and laterally (arrow) (CSTs are in blue); this was the most common pattern of CST displacement in this series, noted in 11 of 13 patients. This pattern of tract displacement makes a transsylvian approach unattractive; one would have to transect the tracts to reach the tumor. D: Postoperative image after an approach through the middle temporal gyrus, using frameless stereotactic navigation to approach the tumor just posterior to the CSTs. Figure is available in color online only. 

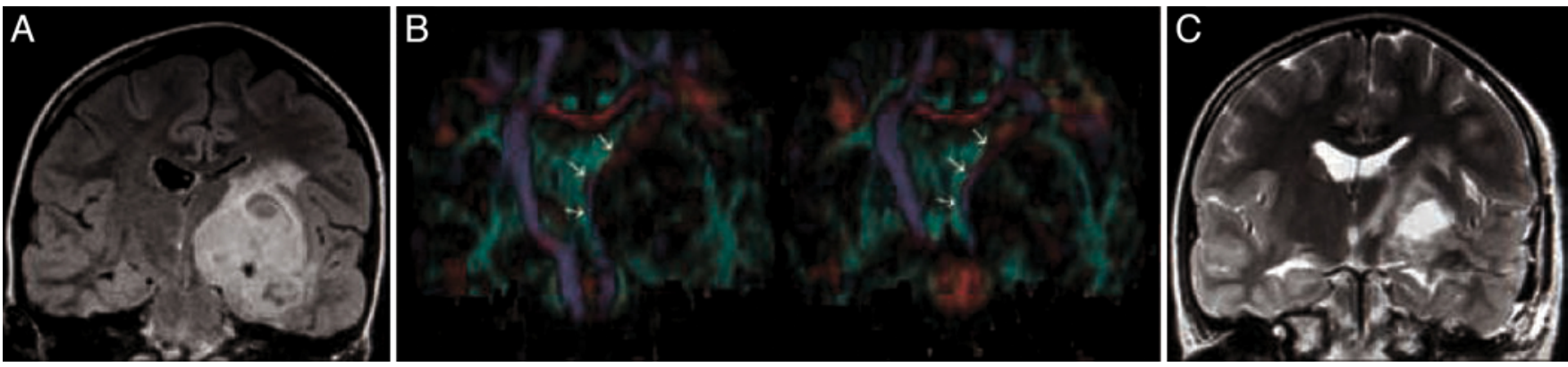

FIG. 2. Left thalamopeduncular cystic and solid pilocytic astrocytoma. A: Coronal FLAIR MR image demonstrating that the tumor grows from the thalamopeduncular junction, across the ambient cistern, and through the choroidal fissure of the temporal horn of the lateral ventricle to invade the middle fossa. B: Coronal DT images showing that the CSTs are displaced medial to the tumor. Medial tract displacement made an image-guided approach through the middle temporal gyrus optimal for reaching both the superior and inferior extent of the neoplasm. C: Coronal T2-weighted MR image obtained the day after surgery, demonstrating the approach through the temporal lobe and GTR of the tumor, sparing the CSTs. Figure is available in color online only.

damaged from our transsylvian approach in Patient 1. There were no further cases of persistent worsening in paresis postoperatively after tractography was implemented in the preoperative planning process. Although patients consistently experienced worsening of their hemiparesis immediately after surgery, with sufficient follow-up they returned to their preoperative status or better.

Intraoperative MRI (iMRI) was available for the last 4 patients in the series. In Patients 10 and 13, iMRI revealed residual tumor after initial resection and allowed for GTR. In Patient 11, a 2-stage surgery was designed up front, knowing that a retromastoid approach would be necessary on second stage to reach portions of the tumor not amenable to a transcortical trans-choroidal approach.

\section{BRAF Status}

Of the 12 tumors tested (not tested in Patient 2 due to insufficient material), 10 (83\%) were positive for the KIAA1549-BRAF fusion (Table 3). None of the 11 tumors tested (2 patients were not tested due to insufficient material) were positive for $B R A F$ V600E point mutation-a mutation that is mutually exclusive to $B R A F$ fusion-by RT-PCR. Of the 2 KIAA1549-BRAF fusion negative tumors, 1 was a unique multifocal tumor that was debulked but could not be cured surgically (Patient 8). In the other, Patient 9, the lesion appeared to be unifocal, as is typical for this series, but had some infiltrative characteristics as well.

\section{Progression and Recurrence}

Follow-up data were obtained for 12 of 13 patients (Table 2). The mean and median times from surgery to most recent follow-up were 50.9 and 33.5 months, respectively (range 4.1-146.9 months). No patients had died of their disease in the follow-up period. Only $2(17 \%)$ of 12 patients required further treatment for progression or recurrence of disease. Patient 11 had progression within the first 2 years and was treated with radiation therapy. The tumor has been stable on imaging for 5 years of follow-up. Patient 13 was noted to have several nodules of recurrence in the original resection cavity approximately 6 months after initial GTR. The recurrence was managed with additional GTR, and the patient is thus far disease free. None of the other 10 patients had recurrence or progression of disease on follow-up, and therefore did not receive any further treatment after surgery. Among those patients with at least 2 years between surgery and most recent imaging, rate of recurrence or progression was also 17\% (1 of 6).

\section{Discussion}

\section{Disease Presentation and Behavior}

The tumors in this series classically present with progressive spastic hemiparesis from mass effect on the CSTs, which has been termed "thalamopeduncular syndrome of childhood." Homonymous hemianopia and ptosis/ophthalmoplegia are less common presenting symptoms that can also occur with respective mass effect on the optic tracts and oculomotor nerves. In our previous report, we

TABLE 3. Genetic characteristics*

\begin{tabular}{ccccc}
\hline $\begin{array}{c}\text { Pt } \\
\text { No.† }\end{array}$ & $\begin{array}{c}\text { Unique } \\
\text { Feature }\end{array}$ & $\begin{array}{c}\text { KIAA1549-BRAF } \\
\text { Fusion }\end{array}$ & $\begin{array}{c}\text { BRAF } \\
\text { V600E }\end{array}$ & PCGP Findings \\
\hline $1(1)$ & - & Positive & Negative & - \\
\hline $2(2)$ & - & - & - & - \\
\hline $3(4)$ & - & Positive & Negative & Negative \\
\hline $4(5)$ & - & Positive & Negative & Negative \\
\hline $5(6)$ & - & Positive & Negative & Negative \\
\hline $6(7)$ & - & Positive & Negative & Negative \\
\hline $7(8)$ & - & Positive & Negative & Negative \\
\hline $8(9)$ & Multifocal & Negative & Negative & - \\
\hline $9(10)$ & Infiltrative & Negative & - & FGFR1-TACC1, \\
& & & & TMPRSS11D:p. \\
& & & & R6S \\
\hline 10 & - & Positive & Negative & - \\
\hline 11 & - & Positive & Negative & - \\
\hline 12 & - & Positive & Negative & - \\
\hline 13 & - & Positive & Negative & - \\
\hline
\end{tabular}

* Molecular genetic features, including presence or absence of KIAA1549$B R A F$ fusion, BRAF V600E point mutation, and additional lesions identified in the PCGP. Both patients with unique clinical features (Patients 8 and 9) were negative for the KIAA1549-BRAF fusion.

$\dagger$ Number in parentheses is case number from previous publication. 
noted headache as a presenting feature in 2 cases. ${ }^{4}$ In 1 of those cases, hydrocephalus was present on preoperative imaging, and symptoms from increased intracranial pressure would likely have arisen had surgery not been performed. In 2 patients whom we have treated since that last publication, symptomatic hydrocephalus was a presenting symptom in addition to hemiparesis. Patient 11 had hydrocephalus that improved with steroid administration. Patient 12 had symptomatic obstructive hydrocephalus that produced nausea and vomiting. He had an especially large tumor that could not be completely resected, and his hydrocephalus was treated with ventricular shunting. Of note, this patient presented from a developing country and had an extended time between neurological symptom onset to surgical intervention. While not a characteristic feature in the presentation of thalamopeduncular tumors, symptomatic hydrocephalus will occur with sufficient tumor progression.

Consistent with pediatric low-grade gliomas (LGGs) elsewhere in the neuraxis, thalamopeduncular tumors are generally not curable with conventional chemotherapy. All 4 tumors in this series treated with chemotherapy progressed and eventually needed definitive surgery, regardless of whether the tumor was previously debulked and subtotally resected. While there exists the possibility of a referral bias such that only the patients whose tumors failed chemotherapy presented to our institution, it is well established that cure rates with conventional chemotherapy in low-grade glioma range from $0 \%$ to $10 \%$.,12,14,27,30 Use of chemotherapy and radiation therapy in treating pediatric LGGs-even when successful-also produces long-term sequelae, including neurocognitive deficits and endocrinopathies. ${ }^{1,12}$ For these reasons, weighed with the advances in preoperative imaging and surgical technique described herein, the treatment priority at our institution is surgical removal-at least until newer agents with favorable side effect profiles are successfully applied to this tumor type.

\section{Surgical Approach and Outcomes}

The results of this case series reflect success in the surgical treatment and cure of pediatric thalamopeduncular tumors. Evaluation of the tumor's relation to adjacent white matter structures is critical in surgical planning, particularly the CSTs, oculomotor nerve, and optic tract. All patients are assessed preoperatively with DTI tractography for this purpose. In a majority of cases, the tumor displaces the CST anterolaterally or medially. For these patients, a transcortical approach through the middle temporal gyrus avoids this structure and allows for a high rate of GTR with minimal complications. The third cranial nerve was injured twice among the 10 patients in whom the middle temporal gyrus approach was used: in one case, it was inadvertently sacrificed because it was indistinguishable from the tumor capsule, and in the other case there was transient palsy secondary to manipulation during resection because the nerve was stretched around the tumor in the ambient cistern. Homonymous hemianopia was a complication in 3 patients - a transsylvian approach was used for 1 case (Patient 2) and a middle temporal gyrus approach in 2 cases. Overall, our rate of persistent neurologi- cal complications from surgery with a transcortical middle temporal gyrus approach was 50\% (5 of 10).

Of the 3 cases of subtotal resection, 2 were not targeted for surgical cure due to unusually large size in one and multifocal nature in the other. In the third case, however, small residual tumor was noted on postoperative imaging. If iMRI had been available for that case, GTR at first surgery might have been possible. In 2 others cases, intraoperative scans revealed residual disease that was then resected to achieve GTR within the same procedure. This modality has great utility in tumor surgery, particularly those cases in which tumors are in deep and surgically challenging locations where the surgeon can err on the side of caution and still achieve GTR when possible.

Only 1 patient (Patient 13) underwent surgery for recurrence. This patient was asymptomatic but had several nodules of local recurrence and a glial cyst noted on MRI that were excised 7 months after initial GTR. On second surgery, near-total resection was achieved, and MRI 9 months later showed a decrease in the area of enhancement. Unfortunately, he suffered homonymous hemianopsia as a consequence of the second surgery. Another patient (Patient 8) required radio- and chemotherapy for progression of atypical multifocal disease 2 years after surgery. As of last contact 5 years later, this patient's disease was stable on imaging. Although he required chemotherapy, radiotherapy, and resection, Patient 8 has been progression free for 5 years, despite having an atypical multifocal tumor. In all, these results argue that aggressive resection of these tumors through the middle temporal gyrus approach provides an excellent rate of disease control.

\section{Molecular Features}

While classification of pediatric LGGs has been largely based on histopathology, this strategy performs poorly for predicting biological behavior. ${ }^{33}$ Recent efforts have focused on molecular characterization, identifying lesions in the mitogen-activated protein kinase (MAPK) pathway as the unifying driver mechanism in pediatric LGGs..$^{18,32,46}$ One such lesion, KIAA1549-BRAF fusion, is recurrent in pediatric pilocytic astrocytomas. ${ }^{6,9,19,23,35,36}$ In the initial results of the PCGP-a whole-genome sequencing collaboration between St. Jude Children's Research Hospital and Washington University in St. Louis-75\% of the 90 pilocytic astrocytomas tested were KIAA1549-BRAF fusion positive ${ }^{46}$ In that series, 42 (89\%) of 45 infratentorial (brainstem and cerebellar) pilocytic astrocytomas were fusion positive, and 21 of 37 (57\%) supratentorial (cerebral cortical and diencephalic) pilocytic astrocytomas were fusion positive. More specifically, brainstem tumors were $80 \%$ positive compared with $59 \%$ of diencephalic lesions.

The high rate of fusion positivity among the thalamopeduncular pilocytic astrocytomas in this series (83\% [10 of 12]) is more consistent with rates seen at an infratentorial or brainstem location. Given also the observed high rate of anterolateral displacement of the CSTs on DTI (83\% [10 of 12]), the molecular and radiological data together suggest a mesencephalic rather than diencephalic origin for this tumor subgroup. Although often classified with thalamic tumors, perhaps thalamopeduncular tumors warrant 
a subclassification of their own and further investigation into their site of origin, which as of now remains unclear. More clearly and consistently sub-classifying these tumors would likely improve academic communication regarding successes and failures in treatment.

Six of the tumors in this series had been previously included in the LGG cohort of the PCGP. ${ }^{46}$ Interestingly, 1 of the 2 thalamopeduncular tumors (Patient 9) negative for KIAA1549-BRAF fusion was found in the PCGP to have a FGFRI-TACCl fusion, an uncommon lesion that substantially activated both MAPK/ERK and PI3K pathways in the tumor. ${ }^{46}$ This patient's tumor was discovered 10 years prior to surgery after a seizure and did not cause appreciable hemiparesis until soon before presentation for surgery. Interestingly, the tumor was noted in the operative report to have superior elements that blended with inferior thalamus parenchyma, suggesting the thalamus as site of origin. Retrospectively, the preoperative imaging also showed some evidence of infiltrative aspects of the lesion, and the pathology report noted features of increased aggressiveness. This tumor also harbored a TMPRSS11D:p. R6S point mutation. None of the other 5 tumors included in the PCGP had significant lesions noted other than KIAA1549-BRAF fusion. The other tumor in our series that was negative for KIAA1549-BRAF fusion (Patient 8) did not have sufficient tissue for any additional molecular testing. This was the unique multifocal tumor that progressed after chemotherapy and surgery and required radiation therapy. The atypical characteristics of these 2 KIAA1549-BRAF fusion-negative cases suggest a correlation between molecular profile and tumor behavior, despite carrying the same histopathological diagnosis and presenting in the same location. However, no conclusions on this can be drawn until a larger sample size is collected.

\section{Implications for Therapy and Future Directions}

These tumors represent a subtype of pediatric LGG that is surgically challenging and has been previously considered incurable or not amenable to GTR. However, with careful planning and the correct approach, these tumors can be cured surgically, in most cases sparing the child from chemotherapy and radiation therapy. In the minority of cases where cure cannot be achieved surgically, the high rate of $B R A F$ fusions and additional MAPK pathway lesions provides an alternative vulnerability for treatment and may replace conventional chemotherapy and radiotherapy. With advances in precision medicine, significant efforts are underway in designing and testing targeted therapies in pediatric LGGs based on the genetic landscape of the individual tumor. The current status of these efforts has been well reviewed recently. ${ }^{33}$ Of relevance, initial trials of selectively targeting BRAF signaling in KIAA1549-BRAF fusion-positive LGGs with traditional selective small molecule inhibitors, like vemurafenib, actually caused paradoxical activation of the MAPK pathway. This is because, in the case of KIAA1549-BRAF, the fusion drives growth by signaling as a constitutive homodimer. A second generation of "paradox-breaking" inhibitors has since been developed and is able to inhibit $B R A F$ fusion signaling in part through disruption of this dimerization. ${ }^{37,45}$ Their application specifically to the
KIAA1549-BRAF fusion and to pediatric LGGs remains to be tested, but offers promise.

At the moment, V600E mutations are the only $B R A F$ lesions being targeted in pediatric LGGs (clinical trial no. PNOC-002). As shown in our series, thalamopeduncular tumors do not display this lesion, as it is characteristic in supratentorial pilocytic astrocytomas, pleomorphic xanthoastrocytomas, and gangliogliomas., ${ }^{9,35}$ However, alternative targets downstream of BRAF are currently being evaluated, such as MEK and MTOR (clinical trial nos. NCT01089101, NCT02124772, NCT00831324, and NCT00955773). ${ }^{21,44}$ Exploiting targets downstream of BRAF will be applicable for thalamopeduncular tumors with less common lesions, like the FGFRl-TACC1 fusion identified in Patient 9, or in cases where a specific lesion cannot be identified but the BRAF pathway is the assumed culprit.

\section{Conclusions}

Pilocytic astrocytomas occurring at the interface of the thalamus and cerebral peduncles have been challenging to manage, both surgically and otherwise. Transcorticaltranschoroidal resection of thalamopeduncular tumors through the middle temporal gyrus allows for a high rate of GTR and disease control with surgical morbidity that is improving as experience is gained. DTI is a critical component of the preoperative planning process to determine the location of white matter tracts in proximity, thus dictating surgical approach. To aid in management, molecular status may correlate with clinical features, and the presence of recurrent $B R A F$ lesions offers an additional target for future novel therapeutics. Because surgical intervention at this anatomically challenging location will always pose some degree of risk, the ideal future approach for these children will require further molecular exploration and subsequent exploitation of individualized targets with agents that have minimal sequelae.

\section{Acknowledgments}

We wish to thank Andrew J. Gienapp (Department of Medical Education, Methodist University Hospital, Memphis, and Department of Neurosurgery, University of Tennessee Health Science Center, Memphis, TN) for technical, copyediting, and publication assistance with this manuscript.

We also wish to sincerely thank Sariah J. Allen, PhD (Department of Pathology, St. Jude Children's Research Hospital, Memphis, TN), for critical assistance with laboratory experiments in defining the molecular characteristics of the tumors, and Ruth G. Tatevossian, MD, PhD (Department of Pathology, St. Jude Children's Research Hospital, Memphis, TN), for assistance with procuring and analyzing relevant genomic data from the Pediatric Cancer Genome Project.

\section{References}

1. Armstrong GT, Conklin HM, Huang S, Srivastava D, Sanford R, Ellison DW, et al: Survival and long-term health and cognitive outcomes after low-grade glioma. Neuro Oncol 13:223-234, 2011

2. Bernstein M, Hoffman HJ, Halliday WC, Hendrick EB, Humphreys RP: Thalamic tumors in children. Long-term followup and treatment guidelines. J Neurosurg 61:649-656, 1984

3. Bouffet E, Jakacki R, Goldman S, Hargrave D, Hawkins C, 
Shroff M, et al: Phase II study of weekly vinblastine in recurrent or refractory pediatric low-grade glioma. J Clin Oncol 30:1358-1363, 2012

4. Broadway SJ, Ogg RJ, Scoggins MA, Sanford R, Patay Z, Boop FA: Surgical management of tumors producing the thalamopeduncular syndrome of childhood. J Neurosurg Pediatr 7:589-595, 2011

5. Carrabba G, Bertani G, Cogiamanian F, Ardolino G, Zarino B, Di Cristofori A, et al: Role of intraoperative neurophysiologic monitoring in the resection of thalamic astrocytomas. World Neurosurg 94:50-56, 2016

6. Cin H, Meyer C, Herr R, Janzarik WG, Lambert S, Jones DT, et al: Oncogenic FAM131B-BRAF fusion resulting from 7q34 deletion comprises an alternative mechanism of MAPK pathway activation in pilocytic astrocytoma. Acta Neuropathol 121:763-774, 2011

7. Cuccia V, Monges J: Thalamic tumors in children. Childs Nerv Syst 13:514-521, 1997

8. DaSilva AF, Tuch DS, Wiegell MR, Hadjikhani N: A primer on diffusion tensor imaging of anatomical substructures. Neurosurg Focus 15(1):E4, 2003

9. Dougherty MJ, Santi M, Brose MS, Ma C, Resnick AC, Sievert AJ, et al: Activating mutations in BRAF characterize a spectrum of pediatric low-grade gliomas. Neuro Oncol 12:621-630, 2010

10. Epstein F: Intrinsic brain stem tumors of childhood, in Kageyama N, Takakura K, Epstein FJ, et al (eds): Intracranial Tumors in Infancy and Childhood. Basel: Karger, 1987, pp 160-169

11. Epstein FJ, Farmer JP: Brain-stem glioma growth patterns. J Neurosurg 78:408-412, 1993

12. Gnekow AK, Falkenstein F, von Hornstein S, Zwiener I, Berkefeld S, Bison B, et al: Long-term follow-up of the multicenter, multidisciplinary treatment study HIT-LGG-1996 for low-grade glioma in children and adolescents of the German Speaking Society of Pediatric Oncology and Hematology. Neuro Oncol 14:1265-1284, 2012

13. Grigsby PW, Thomas PRM, Schwartz HG, Fineberg B: Irradiation of primary thalamic and brainstem tumors in a pediatric population. A 33-year experience. Cancer 60:29012906, 1987

14. Gururangan S, Fisher MJ, Allen JC, Herndon JE II, Quinn JA, Reardon DA, et al: Temozolomide in children with progressive low-grade glioma. Neuro Oncol 9:161-168, 2007

15. Hadjipanayis CG, Kondziolka D, Flickinger JC, Lunsford LD: The role of stereotactic radiosurgery for low-grade astrocytomas. Neurosurg Focus 14(5):e15, 2003

16. Hoffman HJ, Soloniuk DS, Humphreys RP, Drake JM, Becker LE, De Lima BO, et al: Management and outcome of lowgrade astrocytomas of the midline in children: a retrospective review. Neurosurgery 33:964-971, 1993

17. Jenkin RDT, Boesel C, Ertel I, Evans A, Hittle R, Ortega J, et al: Brain-stem tumors in childhood: a prospective randomized trial of irradiation with and without adjuvant CCNU, VCR, and prednisone. A report of the Childrens Cancer Study Group. J Neurosurg 66:227-233, 1987

18. Jones DT, Hutter B, Jäger N, Korshunov A, Kool M, Warnatz HJ, et al: Recurrent somatic alterations of FGFR1 and NTRK2 in pilocytic astrocytoma. Nat Genet 45:927-932, 2013

19. Jones DT, Kocialkowski S, Liu L, Pearson DM, Bäcklund LM, Ichimura K, et al: Tandem duplication producing a novel oncogenic BRAF fusion gene defines the majority of pilocytic astrocytomas. Cancer Res 68:8673-8677, 2008

20. Kelly PJ: Stereotactic biopsy and resection of thalamic astrocytomas. Neurosurgery 25:185-195, 1989

21. Kieran MW, Yao X, Macy M, Leary S, Cohen K, MacDonald T, et al: Final results of a prospective multi-institutional phase ii study of everolimus (RAD001), an mTOR inhibitor, in pediatric patients with recurrent or progressive low-grade glioma. A POETIC consortium trial. Neuro Oncol 16:iii27, 2014 (Abstract)

22. Kis D, Máté A, Kincses ZT, Vörös E, Barzó P: The role of probabilistic tractography in the surgical treatment of thalamic gliomas. Neurosurgery 10 (Suppl 2):262-272, 2014

23. Lin A, Rodriguez FJ, Karajannis MA, Williams SC, Legault $\mathrm{G}$, Zagzag D, et al: BRAF alterations in primary glial and glioneuronal neoplasms of the central nervous system with identification of 2 novel KIAA1549:BRAF fusion variants. J Neuropathol Exp Neurol 71:66-72, 2012

24. McGirr SJ, Kelly PJ, Scheithauer BW: Stereotactic resection of juvenile pilocytic astrocytomas of the thalamus and basal ganglia. Neurosurgery 20:447-452, 1987

25. Moshel YA, Elliott RE, Monoky DJ, Wisoff JH: Role of diffusion tensor imaging in resection of thalamic juvenile pilocytic astrocy toma. J Neurosurg Pediatr 4:495-505, 2009

26. Moshel YA, Link MJ, Kelly PJ: Stereotactic volumetric resection of thalamic pilocytic astrocytomas. Neurosurgery 61:66-75, 2007

27. Packer RJ, Ater J, Allen J, Phillips P, Geyer R, Nicholson HS, et al: Carboplatin and vincristine chemotherapy for children with newly diagnosed progressive low-grade gliomas. J Neurosurg 86:747-754, 1997

28. Phillips NS, Sanford RA, Helton KJ, Boop FA, Zou P, Tekautz T, et al: Diffusion tensor imaging of intraaxial tumors at the cervicomedullary and pontomedullary junctions. Report of two cases. J Neurosurg 103 (6 Suppl):557-562, 2005

29. Pierre-Kahn A, Hirsch JF, Vinchon M, Payan C, Sainte-Rose C, Renier D, et al: Surgical management of brain-stem tumors in children: results and statistical analysis of 75 cases. $\mathbf{J}$ Neurosurg 79:845-852, 1993

30. Prados MD, Edwards MS, Rabbitt J, Lamborn K, Davis RL, Levin VA: Treatment of pediatric low-grade gliomas with a nitrosourea-based multiagent chemotherapy regimen. J Neurooncol 32:235-241, 1997

31. Puget S, Crimmins DW, Garnett MR, Grill J, Oliveira R, Boddaert N, et al: Thalamic tumors in children: a reappraisal. J Neurosurg 106 (5 Suppl):354-362, 2007

32. Ramkissoon LA, Horowitz PM, Craig JM, Ramkissoon SH, Rich BE, Schumacher SE, et al: Genomic analysis of diffuse pediatric low-grade gliomas identifies recurrent oncogenic truncating rearrangements in the transcription factor MYBL1. Proc Natl Acad Sci U S A 110:8188-8193, 2013

33. Robinson GW, Witt H, Resnick A: Exploiting laboratory insights to improve outcomes of pediatric central nervous system tumors. Am Soc Clin Oncol Educ Book 35:e540-e546, 2016

34. Sai Kiran NA, Thakar S, Dadlani R, Mohan D, Furtado SV, Ghosal N, et al: Surgical management of thalamic gliomas: case selection, technical considerations, and review of literature. Neurosurg Rev 36:383-393, 2013

35. Schindler G, Capper D, Meyer J, Janzarik W, Omran H, Herold-Mende C, et al: Analysis of BRAF V600E mutation in 1,320 nervous system tumors reveals high mutation frequencies in pleomorphic xanthoastrocytoma, ganglioglioma and extra-cerebellar pilocytic astrocytoma. Acta Neuropathol 121:397-405, 2011

36. Sievert AJ, Jackson EM, Gai X, Hakonarson H, Judkins AR, Resnick AC, et al: Duplication of 7q34 in pediatric low-grade astrocytomas detected by high-density single-nucleotide polymorphism-based genotype arrays results in a novel BRAF fusion gene. Brain Pathol 19:449-458, 2009

37. Sievert AJ, Lang SS, Boucher KL, Madsen PJ, Slaunwhite E, Choudhari N, et al: Paradoxical activation and RAF inhibitor resistance of BRAF protein kinase fusions characterizing pediatric astrocytomas. Proc Natl Acad Sci U S A 110:5957-5962, 2013 
38. Souweidane MM, Hoffman HJ: Current treatment of thalamic gliomas in children. J Neurooncol 28:157-166, 1996

39. Steinbok P, Gopalakrishnan CV, Hengel AR, Vitali AM, Poskitt K, Hawkins C, et al: Pediatric thalamic tumors in the MRI era: a Canadian perspective. Childs Nerv Syst 32:269280, 2016

40. Stroink AR, Hoffman HJ, Hendrick EB, Humphreys RP: Diagnosis and management of pediatric brain-stem gliomas. $\mathbf{J}$ Neurosurg 65:745-750, 1986

41. Tomita T, Cortes RF: Astrocytomas of the cerebral peduncle in children: surgical experience in seven patients. Childs Nerv Syst 18:225-230, 2002

42. Tovi D, Schisano G, Liljeqvist B: Primary tumors of the region of the thalamus. J Neurosurg 18:730-740, 1961

43. Vandertop WP, Hoffman HJ, Drake JM, Humphreys RP, Rutka JT, Amstrong DC, et al: Focal midbrain tumors in children. Neurosurgery 31:186-194, 1992

44. Yalon M, Rood B, MacDonald TJ, McCowage G, Kane R, Constantini S, et al: A feasibility and efficacy study of rapamycin and erlotinib for recurrent pediatric low-grade glioma (LGG). Pediatr Blood Cancer 60:71-76, 2013

45. Zhang C, Spevak W, Zhang Y, Burton EA, Ma Y, Habets G, et al: RAF inhibitors that evade paradoxical MAPK pathway activation. Nature 526:583-586, 2015

46. Zhang J, Wu G, Miller CP, Tatevossian RG, Dalton JD, Tang $\mathrm{B}$, et al: Whole-genome sequencing identifies genetic altera- tions in pediatric low-grade gliomas. Nat Genet 45:602-612, 2013

47. Zheng X, Xu X, Zhang H, Wang Q, Ma X, Chen X, et al: A preliminary experience with use of intraoperative magnetic resonance imaging in thalamic glioma surgery: a case series of 38 patients. World Neurosurg 89:434-441, 2016

\section{Disclosures}

Dr. Boop has served as a consultant for Medtronic.

\section{Author Contributions}

Conception and design: Lee, Foster, Orr. Acquisition of data: Lee, Foster. Analysis and interpretation of data: Boop, Lee, Foster, Lillard, Ellison, Orr. Drafting the article: Boop, Lee. Critically revising the article: Boop, Lee, Lillard, Klimo. Reviewed submitted version of manuscript: Boop, Lee. Approved the final version of the manuscript on behalf of all authors: Boop. Statistical analysis: Lee. Study supervision: Boop, Klimo, Ellison, Orr.

\section{Correspondence}

Frederick A. Boop, Semmes-Murphey Neurologic \& Spine Institute, 6325 Humphreys Blvd., Memphis, TN 38120. email: frederickboop@gmail.com. 